\title{
Normal approximation for call function of locally dependent random variables
}

\author{
Suporn Jongpreechaharn ${ }^{\mathrm{a}}$, Kritsana Neammanee ${ }^{\mathrm{a}, \mathrm{b}, *}$ \\ a Department of Mathematics and Computer Science, Faculty of Science, Chulalongkorn University, \\ Bangkok 10330 Thailand \\ b Centre of Excellence in Mathematics, Commission on Higher Education, Bangkok, Thailand \\ *Corresponding author, e-mail: kritsana.n@chula.ac.th
}

Received 7 Jun 2021

Accepted 29 Nov 2021

\begin{abstract}
A mean for the call function of random variable $W, E(W-k)^{+}$, where $k$ is a positive real number, is useful and important, for instance, in a collateralized debt obligation (CDO) tranche pricing. In previous works, $E(W-k)^{+}$ is approximated by normal approximation where $W$ is a sum of independent random variables. However, $W$ in this paper is extended to be a sum of locally dependent random variables. We propose a uniform bound on the normal approximation by using the Stein's method.
\end{abstract}

KEYWORDS: normal approximation, call function, local dependence, Stein's method, uniform bound

MSC2020: 60F05

\section{INTRODUCTION}

A collateralized debt obligation (CDO) is a financial asset inducing an enormous crisis named the Hamburger crisis between 2007 and 2008. After that, many researchers have attempted to manage the risk in CDO. For example, the factor model [1-3], the saddle point method [4] and the dual quantization method [5]. In addition, an approximation approach dealing with a mean for a call function of the random variable $W$ and a fixed real number $k$, represented a CDO tranche price,

$$
E(W-k)^{+}
$$

was concentrated $[6,7]$ where $(a)^{+}=\max \{a, 0\}$ for any $a \in \mathbb{R}$. The Stein's method is used to approximate a mean for a call function by normal and Poisson approximations where $W$ is a sum of independent random variables. The results were refined by Yonghint and Neammanee [8] (see also $[9,10]$ ) and Jongpreechaharn and Neammanee [11] under the same assumptions.

Apart from independence of random variables, Chen and Shao [12] introduced a general local dependence. They proposed various bounds for Kolmogorov distance,

$$
\sup _{z \in \mathbb{R}}|\operatorname{Pr}(W \leqslant z)-\Phi(z)|,
$$

where $W$ is a sum of locally dependent random variables and $\Phi$ is the standard normal distribution function. The dependence condition has extended to many features. Under various types of local dependence, sums of Bernoulli $[9,13]$, integer-valued [14] or realvalued [15] random variables are approximated by Poisson distribution, compound Poisson distribution and Poisson process. In addition, a centered and symmetric binomial random variable [16] and pseudobinomial and negative binomial random variables [17] are also used in approximation. Moreover, discretized normal [18], multidimensional normal [19] and multivariate discrete normal [20] distributions are considered.

In this work, we adopt a local dependence assumption from $[12]$ to extend the results of $[6,7,11]$. Let $X_{1}, X_{2}, \ldots, X_{n}$ be zero means and finite variances random variables. Denote

$$
W=\sum_{i=1}^{n} X_{i}
$$

and assume that $W$ has a unit variance. Suppose that $X_{1}, X_{2}, \ldots, X_{n}$ satisfy the local dependence defined as follows.

For $A \subseteq\{1,2, \ldots, n\}$, let $X_{A}$ denotes $\left\{X_{i}: i \in A\right\}$ and $X_{A^{c}}=\left\{X_{i}: i \notin A\right\}$. We say that $X_{1}, X_{2}, \ldots, X_{n}$ satisfy the local dependence condition if there exists a partition $\left\{A_{i}\right\}_{i=1}^{l_{n}}$ of $\{1,2, \ldots, n\}$ such that for each $\left\{1,2, \ldots, l_{n}\right\}, X_{A_{i}}$ is independent of $X_{A_{i}^{c}}$. It means that, we can categorize random variables into groups such that random variables in the same group may be correlated whereas random variables from different groups are independent. For instance, in a CDO, we can classify assets from a source of their revenue because default occurs when debtors are having a cash-flow problem. Suppose that the CDO contains indebted creator and account executive working in the same company, orchardist, fruit seller and fruit processing factory owner (Fig. 1). We put two office workers in the same group because they may simultaneously default, if the company takes a pay cut or goes bankruptcy. Additionally, orchardist, fruit seller and fruit processing factory owner belong to a group 


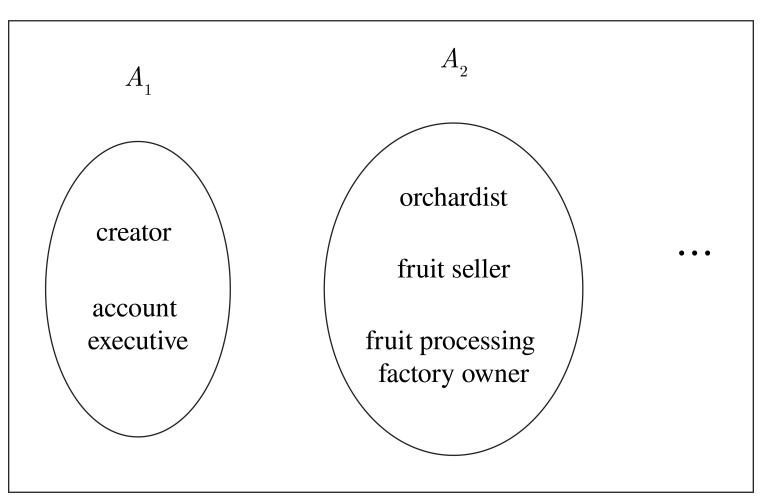

Fig. 1 Example of classification for locally dependent assets in a CDO.

because of their common product. If the orchardist can not produce fruit, then fruit seller and fruit processing factory owner may be disturbed. Meanwhile, fruit seller and creator are independent.

From now on, we assume that $X_{1}, X_{2}, \ldots, X_{n}$ are locally dependent. Let $Z$ be a standard normal random variable. From the above setting, we use the Stein's method to obtain a uniform bound for

$$
\left|E(W-k)^{+}-E(Z-k)^{+}\right|
$$

under local dependence. The following is the main result.

Theorem 1 For each $i=1,2, \ldots, l_{n}$, let $Y_{i}=\sum_{j \in A_{i}} X_{j}$. Under the local dependence condition and $k>0$, we have

$$
\begin{gathered}
\sup _{k>0}\left|E(W-k)^{+}-E(Z-k)^{+}\right| \leqslant 24.97 \sum_{i=1}^{l_{n}} E\left|Y_{i}\right|^{3}+ \\
0.80\left(\sum_{i=1}^{l_{n}} E Y_{i}^{4}\right)^{1 / 2}+\left(l_{n} E W^{4} \sum_{i=1}^{l_{n}} E Y_{i}^{6}\right)^{1 / 2},
\end{gathered}
$$

where $E W^{4} \leqslant 3+\sum_{i=1}^{l_{n}} E Y_{i}^{4}$. Furthermore, if $Y_{i}=$ $O\left(\frac{1}{\sqrt{n}}\right)$ and $l_{n}=O(n)$, then

$$
\sup _{k>0}\left|E(W-k)^{+}-E(Z-k)^{+}\right|=O\left(\frac{1}{\sqrt{n}}\right) .
$$

\section{STEIN'S METHOD FOR CALL FUNCTION}

In this section, we introduce a brilliant method for obtaining a bound on the normal approximation discovered by Stein [21] in 1972, called the Stein's method. We also give a useful property of the Stein solution for the call function.

Let $Z$ be a standard normal random variable and $f: \mathbb{R} \rightarrow \mathbb{R}$ be an absolutely continuous function with $E\left|f^{\prime}(Z)\right|<\infty$. The Stein's method begins with the characterization of $Z$,

$$
E Z f(Z)=E f^{\prime}(Z)
$$

From this equation, we have the Stein equation on a normal approximation for a given function $h$ as

$$
x f(x)-f^{\prime}(x)=h(x)-E h(Z) .
$$

In this work, we apply the Stein equation (1) with the call function $h(x)=(x-k)^{+}$where $k>0$ providing

$$
x f_{k}(x)-f_{k}^{\prime}(x)=(x-k)^{+}-E(Z-k)^{+}
$$

where $f_{k}$ is the solution of (1) in the case that $h(x)=$ $(x-k)^{+}$. Therefore, for any random variable $W$, we have

$$
E W f_{k}(W)-E f_{k}^{\prime}(W)=E(W-k)^{+}-E(Z-k)^{+} .
$$

From this equation, we determine an error bound of $\left|E W f_{k}(W)-E f_{k}^{\prime}(W)\right|$ instead of $\left|E(W-k)^{+}-E(Z-k)^{+}\right|$ which is an important technique in the Stein's method.

Moreover, the explicit Stein solution $f_{k}$ and its derivative play an important role in this work. We use the argument given by $[11,21]$ to obtain

$$
f_{k}(x)= \begin{cases}\sqrt{2 \pi} \mathrm{e}^{x^{2} / 2} E(Z-k)^{+} \Phi(x), & x \leqslant k, \\ 1-\sqrt{2 \pi} \mathrm{e}^{x^{2} / 2}\left[k+E(Z-k)^{+}\right] \Phi(-x), & x>k\end{cases}
$$

and

$f_{k}^{\prime}(x)= \begin{cases}E(Z-k)^{+}\left(1+\sqrt{2 \pi} x \Phi(x) \mathrm{e}^{x^{2} / 2}\right), & x \leqslant k, \\ {\left[k+E(Z-k)^{+}\right]\left(1-\sqrt{2 \pi} x \Phi(-x) \mathrm{e}^{x^{2} / 2}\right),} & x>k,\end{cases}$

where $\Phi$ is the cumulative distribution function of $Z$.

The next proposition is used to prove the main result.

Proposition 1 For real numbers $x, t$ with $|t| \leqslant 1$ and $a$ positive real number $k$, we have

$$
\left|f_{k}^{\prime}(x+t)-f_{k}^{\prime}(x)\right| \leqslant 2 x^{2}|t|+10.46|x||t|+12.16|t| .
$$

Proof: From (2), we have

$$
\begin{aligned}
& f_{k}^{\prime}(x+t)-f_{k}^{\prime}(x) \\
& =(x+t) f_{k}(x+t)-x f_{k}(x)-(x+t-k)^{+}+(x-k)^{+} \\
& = \begin{cases}(x+t) f_{k}(x+t)-x f_{k}(x)-t ; & x>k, x+t>k, \\
(x+t) f_{k}(x+t)-x f_{k}(x) ; & x \leqslant k, x+t \leqslant k, \\
(x+t) f_{k}(x+t)-x f_{k}(x)+(x-k) ; & x>k, x+t \leqslant k, \\
(x+t) f_{k}(x+t)-x f_{k}(x)-(x+t-k) ; & x \leqslant k, x+t>k .\end{cases}
\end{aligned}
$$

Case 1: $x>k$ and $x+t>k$. From [22] (Lemma 2.4, p.16), we have that

$$
\left\|f_{k}\right\| \leqslant 2
$$

and

$$
\left\|f_{k}^{\prime}\right\| \leqslant \sqrt{\frac{2}{\pi}} \leqslant 0.8
$$


where $\|g\|=\sup _{x \in \mathbb{R}}|g(x)|$ for any real-valued function $g$ on $\mathbb{R}$. Since $f_{k}$ is continuous on $(k, \infty)$, we use the mean value theorem, (6) and (7) to show that

$$
\begin{aligned}
\mid f_{k}^{\prime}(x & +t)-f_{k}^{\prime}(x) \mid \\
& =\left|x\left[f_{k}(x+t)-f_{k}(x)\right]+t f_{k}(x+t)-t\right| \\
& \leqslant|x|\left|f_{k}(x+t)-f_{k}(x)\right|+|t|\left(\left|f_{k}(x+t)\right|+1\right) \\
& \leqslant|x||| f_{k}^{\prime} \||t|+3|t| \\
& \leqslant 0.8|x||t|+3|t| .
\end{aligned}
$$

Case 2: $x \leqslant k$ and $x+t \leqslant k$. By using the same argument shown in Case 1 with the fact that $f_{k}$ is continuous on $(-\infty, k]$, we conclude that

$$
\begin{aligned}
\mid f_{k}^{\prime}(x+t)- & f_{k}^{\prime}(x) \mid \\
& \leqslant|x|\left|f_{k}(x+t)-f_{k}(x)\right|+|t|\left|f_{k}(x+t)\right| \\
& \leqslant 0.8|x||t|+2|t| .
\end{aligned}
$$

Case 3: $k<x \leqslant k-t$. By (3) and (5), we have

$$
\begin{aligned}
f_{k}^{\prime}(x+t)-f_{k}^{\prime}(x) \\
=(x+t) f_{k}(x+t)-x f_{k}(x)+x-k \\
=\sqrt{2 \pi}(x+t) \mathrm{e}^{(x+t)^{2} / 2} E(Z-k)^{+} \Phi(x+t) \\
+\sqrt{2 \pi} x \mathrm{e}^{x^{2} / 2}\left[k+E(Z-k)^{+}\right] \Phi(-x)-k .
\end{aligned}
$$

Note that

$$
E(Z-k)^{+}=\frac{\mathrm{e}^{-k^{2} / 2}}{\sqrt{2 \pi}}-k \Phi(-k)=\frac{\mathrm{e}^{-k^{2} / 2}}{\sqrt{2 \pi}}+k \Phi(k)-k .
$$

This implies that

$$
\begin{gathered}
\sqrt{2 \pi}(x+t) \mathrm{e}^{(x+t)^{2} / 2} E(Z-k)^{+} \Phi(x+t) \\
=\sqrt{2 \pi}(x+t) \mathrm{e}^{(x+t)^{2} / 2} \Phi(x+t)\left[\frac{\mathrm{e}^{-k^{2} / 2}}{\sqrt{2 \pi}}+k \Phi(k)\right] \\
-\sqrt{2 \pi} k(x+t) \mathrm{e}^{(x+t)^{2} / 2} \Phi(x+t) .
\end{gathered}
$$

By (9) and the fact that $\Phi(x+t)=1-\Phi(-(x+t))$, we obtain

$$
\begin{aligned}
& \sqrt{2 \pi}(x+t) \mathrm{e}^{(x+t)^{2} / 2} \Phi(x+t)\left[\frac{\mathrm{e}^{-k^{2} / 2}}{\sqrt{2 \pi}}+k \Phi(k)\right] \\
& =\sqrt{2 \pi}(x+t) \mathrm{e}^{(x+t)^{2} / 2}\left[\frac{\mathrm{e}^{-k^{2} / 2}}{\sqrt{2 \pi}}+k \Phi(k)\right] \\
& -\sqrt{2 \pi}(x+t) \mathrm{e}^{(x+t)^{2} / 2} \Phi(-(x+t))\left[k+E(Z-k)^{+}\right] .
\end{aligned}
$$

Combining (8), (10) and (11), we obtain

$$
f_{k}^{\prime}(x+t)-f_{k}^{\prime}(x)=B_{1}+B_{2}+B_{3}
$$

where

$$
\begin{aligned}
B_{1} & =(x+t) \mathrm{e}^{(x+t)^{2} / 2-k^{2} / 2}-k, \\
B_{2} & =(x+t) \sqrt{2 \pi} k \mathrm{e}^{(x+t)^{2} / 2}[\Phi(k)-\Phi(x+t)], \\
B_{3} & =\sqrt{2 \pi}\left[k+E(Z-k)^{+}\right]\left[g_{1}(x)-g_{1}(x+t)\right], \\
g_{1}(s) & =s \mathrm{e}^{s^{2} / 2} \Phi(-s) .
\end{aligned}
$$

First, we consider $B_{1}$. If $x+t<0$, then $(x+t)$ $\mathrm{e}^{(x+t)^{2} / 2-k^{2} / 2} \leqslant 0$. If $x+t \geqslant 0$, then $0 \leqslant x+t \leqslant k$. Thus, $(x+t) \mathrm{e}^{(x+t)^{2} / 2-k^{2} / 2} \leqslant x+t$. These imply that

$$
B_{1}=(x+t) \mathrm{e}^{(x+t)^{2} / 2-k^{2} / 2}-k \leqslant(x+t)-k \leqslant 0 .
$$

To find a lower bound for $B_{1}$, we consider possible values of $x+t$ including $-k<x+t \leqslant 0,-1 \leqslant x+t \leqslant$ $-k$ and $0<x+t \leqslant k$.

If $-k<x+t \leqslant 0$, then $B_{1} \geqslant x+t-k>-|t|$. Thus,

$$
-|t|<B_{1} \leqslant 0 \text { for }-k<x+t \leqslant 0 .
$$

If $-1 \leqslant x+t \leqslant-k$, then $(x+t)^{2}-k^{2} \geqslant 0$ and $k \leqslant 1$. Note by the mean value theorem that for any $a \in \mathbb{R}$,

$$
\mathrm{e}^{a}=1+a \mathrm{e}^{b}
$$

for some $\min \{a, 0\}<b<\max \{a, 0\}$. By (13), we have

$$
\mathrm{e}^{(x+t)^{2} / 2-k^{2} / 2}=1+\left(\frac{(x+t)^{2}}{2}-\frac{k^{2}}{2}\right) \mathrm{e}^{x_{0}}
$$

for some $0 \leqslant x_{0} \leqslant \frac{(x+t)^{2}}{2}-\frac{k^{2}}{2}$. By using inequalities $-1 \leqslant x+t+k \leqslant 0$ and $-1 \leqslant t<x+t-k \leqslant 0$, we have $0 \leqslant(x+t)^{2}-k^{2} \leqslant|t| \leqslant 1$. Hence,

$$
\begin{aligned}
B_{1} & =(x+t)\left[1+\left(\frac{(x+t)^{2}}{2}-\frac{k^{2}}{2}\right) \mathrm{e}^{x_{0}}\right]-k \\
& \geqslant x+t+\frac{\sqrt{\mathrm{e}}(x+t)|t|}{2}-k \\
& \geqslant-|t|-\frac{\sqrt{\mathrm{e}}|t|}{2} \\
& \geqslant-1.83|t| \quad \text { for }-1 \leqslant x+t \leqslant-k .
\end{aligned}
$$

Therefore,

$$
-1.83|t| \leqslant B_{1} \leqslant 0 \text { for }-1 \leqslant x+t \leqslant-k .
$$

Suppose that $0<x+t \leqslant k$. Then, $\frac{(x+t)^{2}}{2}-\frac{k^{2}}{2}<0$. By (13), we have

$$
\mathrm{e}^{\frac{(x+t)^{2}}{2}-\frac{k^{2}}{2}}=1+\left(\frac{(x+t)^{2}}{2}-\frac{k^{2}}{2}\right) \mathrm{e}^{x_{1}}
$$

for some $x_{1}<0$. Note that $\frac{(x+t)^{2}}{2}-\frac{k^{2}}{2}=\frac{x^{2}}{2}+x t+\frac{t^{2}}{2}-$ $\frac{k^{2}}{2} \geqslant x t+\frac{t^{2}}{2}$. Hence,

$$
\begin{aligned}
B_{1} & =(x+t)\left[1+\left(\frac{(x+t)^{2}}{2}-\frac{k^{2}}{2}\right) \mathrm{e}^{x_{1}}\right]-k \\
& \geqslant x+t+(x+t)\left(x t+\frac{t^{2}}{2}\right)-k \\
& \geqslant-|t|-x^{2}|t|-\frac{|t|^{3}}{2} \\
& \geqslant-x^{2}|t|-1.5|t| \quad \text { for } 0<x+t \leqslant k .
\end{aligned}
$$


Thus,

$$
-x^{2}|t|-1.5|t| \leqslant B_{1} \leqslant 0 \quad \text { for } 0<x+t \leqslant k .
$$

By (12)-(15), we obtain

$$
\left|B_{1}\right| \leqslant x^{2}|t|+1.83|t| \text { for } k<x \leqslant k-t .
$$

Next, we consider

$$
B_{2}=(x+t) \sqrt{2 \pi} k \mathrm{e}^{(x+t)^{2} / 2}[\Phi(k)-\Phi(x+t)]
$$

for $k<x \leqslant k-t$.

If $x+t<0$, then $B_{2}<0$. Notice that, there exists $c \in(x+t, k)$ such that

$$
\Phi(k)-\Phi(x+t)=\Phi^{\prime}(c)(k-x-t) \leqslant \frac{|t|}{\sqrt{2 \pi}} .
$$

Since $x+t<0, k<x<-t \leqslant 1$, by this inequality and $-1 \leqslant t<k+t<x+t<0$, we have

$$
B_{2} \geqslant(x+t) k \mathrm{e}^{(x+t)^{2} / 2}|t|>-\sqrt{\mathrm{e}}|t| \geqslant-1.65|t|
$$

for $x+t<0$. Then,

$$
-1.65|t| \leqslant B_{2}<0 \quad \text { for } x+t<0 \text {. }
$$

Suppose that $x+t \geqslant 0$. Then, $B_{2} \geqslant 0$. Note that for $0 \leqslant a \leqslant b$,

$$
\Phi(b)-\Phi(a)=\frac{1}{\sqrt{2 \pi}} \int_{a}^{b} \mathrm{e}^{-s^{2} / 2} \mathrm{~d} s \leqslant \frac{\mathrm{e}^{-a^{2} / 2}}{\sqrt{2 \pi}}(b-a) .
$$

From (16) and the inequality $x+t \leqslant k<x$, we have $0 \leqslant B_{2} \leqslant(x+t) k|t| \leqslant k x|t| \leqslant x^{2}|t|$ for $x+t \geqslant 0$. Hence,

$$
\left|B_{2}\right| \leqslant x^{2}|t|+1.65|t| \quad \text { for } x+t \in \mathbb{R} .
$$

To bound $B_{3}=\sqrt{2 \pi}\left(k+E(Z-k)^{+}\right)\left[g_{1}(x)-g_{1}(x+\right.$ $t)]$ where $g_{1}(s)=s \mathrm{e}^{s^{2} / 2} \Phi(-s)$, we first show that

$$
\left|g_{1}^{\prime}(s)\right| \leqslant 3.18 \text { for } s \geqslant-1 .
$$

Note that $g_{1}^{\prime}(s)=-\frac{s}{\sqrt{2 \pi}}+\Phi(-s) \mathrm{e}^{s^{2} / 2}\left(s^{2}+1\right)$. If $|s| \leqslant 1$, then $\left|g_{1}^{\prime}(s)\right| \leqslant \frac{1}{\sqrt{2 \pi}}+2 \Phi(1) \mathrm{e}^{1 / 2} \leqslant 3$.18. Suppose that $s>1$. For $a>0$, we have $\Phi(-a) \leqslant \frac{\mathrm{e}^{-a^{2} / 2}}{\sqrt{2 \pi} a}$ (see [23], p.23) and $\Phi(-a) \geqslant \frac{\mathrm{e}^{-a^{2} / 2}}{\sqrt{2 \pi}}\left(\frac{1}{a}-\frac{1}{a^{3}}\right)$ (see [11], p.3502). These imply that $\left|g_{1}^{\prime}(s)\right| \leqslant \frac{1}{\sqrt{2 \pi}}$. Hence,

$$
\left|g_{1}^{\prime}(s)\right| \leqslant 3.18 \text { for } s \geqslant-1 .
$$

Notice that $g_{1}(x)-g_{1}(x+t)=-t g_{1}^{\prime}(c)$ for some $x+t<$ $c<x$. From this fact, (17) and the fact that

$$
E(Z-k)^{+} \leqslant \frac{\mathrm{e}^{-k^{2} / 2}}{\sqrt{2 \pi}} \quad(\text { see }[11], \mathrm{p} .3502),
$$

we have

$$
\left|B_{3}\right| \leqslant \sqrt{2 \pi}\left(x+\frac{1}{\sqrt{2 \pi}}\right)\left|g_{1}^{\prime}(c)\right||t| \leqslant 7.98|x||t|+3.18|t| .
$$

Consequently,

$$
\left|f_{k}^{\prime}(x+t)-f_{k}^{\prime}(x)\right| \leqslant 2 x^{2}|t|+7.98|x||t|+6.66|t|
$$

for $k<x \leqslant k-t$.

Case 4: $k-t<x \leqslant k$. By (3) and (5), we have

$$
\begin{aligned}
& f_{k}^{\prime}(x+t)-f_{k}^{\prime}(x) \\
& =(x+t) f_{k}(x+t)-x f_{k}(x)-(x+t-k) \\
& =-\sqrt{2 \pi}(x+t) \mathrm{e}^{(x+t)^{2} / 2}\left[k+E(Z-k)^{+}\right] \Phi(-(x+t)) \\
& \quad-\sqrt{2 \pi} x \mathrm{e}^{x^{2} / 2} E(Z-k)^{+} \Phi(x)+k .
\end{aligned}
$$

By (9), we have

$$
\begin{array}{r}
-\sqrt{2 \pi} x \mathrm{e}^{x^{2} / 2} E(Z-k)^{+} \Phi(x)=\sqrt{2 \pi} x \mathrm{e}^{x^{2} / 2} k \Phi(x) \\
-\sqrt{2 \pi} x \mathrm{e}^{x^{2} / 2}\left[\frac{\mathrm{e}^{-k^{2} / 2}}{\sqrt{2 \pi}}+k \Phi(k)\right] \Phi(x) .
\end{array}
$$

By (9) and the fact that $\Phi(x)=1-\Phi(-x)$, we have

$$
\begin{aligned}
-\sqrt{2 \pi} x & \mathrm{e}^{x^{2} / 2}\left[\frac{\mathrm{e}^{-k^{2} / 2}}{\sqrt{2 \pi}}+k \Phi(k)\right] \Phi(x) \\
= & -\sqrt{2 \pi} x \mathrm{e}^{x^{2} / 2}\left[\frac{\mathrm{e}^{-k^{2} / 2}}{\sqrt{2 \pi}}+k \Phi(k)\right] \\
& +\sqrt{2 \pi} x \mathrm{e}^{x^{2} / 2}\left[k+E(Z-k)^{+}\right] \Phi(-x) .
\end{aligned}
$$

Combining (19)-(21), we obtain

$$
f_{k}^{\prime}(x+t)-f_{k}^{\prime}(x)=C_{1}+C_{2}+C_{3},
$$

where

$$
\begin{aligned}
& C_{1}=\sqrt{2 \pi}\left[k+E(Z-k)^{+}\right]\left[g_{1}(x)-g_{1}(x+t)\right], \\
& C_{2}=\sqrt{2 \pi} k x \mathrm{e}^{x^{2} / 2}[\Phi(x)-\Phi(k)] \\
& C_{3}=-x \mathrm{e}^{x^{2} / 2-k^{2} / 2}+k .
\end{aligned}
$$

By (17), (18) and the inequality $0<k<x+t \leqslant x+1$, we have

$$
\begin{aligned}
\left|C_{1}\right| & \leqslant \sqrt{2 \pi}\left(|x|+1+\frac{1}{\sqrt{2 \pi}}\right)\left|g_{1}^{\prime}\left(s_{0}\right)\right||t| \\
& \leqslant 7.98|x||t|+11.16|t|
\end{aligned}
$$

for some $s_{0} \in(x, x+t) \subseteq[-1, \infty)$.

Next, we consider $C_{2}$. If $x \geqslant 0$, then $C_{2} \leqslant 0$. By (16), we have

$$
\begin{aligned}
-C_{2} & =\sqrt{2 \pi} k x \mathrm{e}^{x^{2} / 2}[\Phi(k)-\Phi(x)] \\
& \leqslant k x(k-x) \\
& \leqslant(|x|+1)|x||t| \\
& =x^{2}|t|+|x||t| .
\end{aligned}
$$


Hence,

$$
-x^{2}|t|-|x||t| \leqslant C_{2} \leqslant 0 \quad \text { for } x \geqslant 0
$$

Suppose that $x<0$. Then, $C_{2}>0$. Since $k-t<$ $x<0, k<t \leqslant 1$. By this inequality and $-1<k-t<$ $x<0$, we have

$$
\begin{aligned}
C_{2} & =k x \mathrm{e}^{x^{2} / 2} \int_{k}^{x} \mathrm{e}^{-s^{2} / 2} \mathrm{~d} s=k|x| \mathrm{e}^{x^{2} / 2} \int_{x}^{k} \mathrm{e}^{-s^{2} / 2} \mathrm{~d} s \\
& \leqslant|x| \mathrm{e}^{x^{2} / 2}(k-x) \leqslant \sqrt{\mathrm{e}}|x||t| \leqslant 1.65|x||t| .
\end{aligned}
$$

Hence,

$$
0<C_{2} \leqslant 1.65|x||t| \quad \text { for } x<0 \text {. }
$$

By (22) and (23), we obtain

$$
\left|C_{2}\right| \leqslant x^{2}|t|+1.65|x||t| \quad \text { for } x \in \mathbb{R}
$$

Finally, we consider $C_{3}=-x e^{x^{2} / 2-k^{2} / 2}+k$ for $k-$ $t<x \leqslant k$. If $x \leqslant 0$, then $C_{3} \geqslant 0$. If $x>0$, then $0<$ $x \leqslant k$. This implies that $C_{3} \geqslant-x+k \geqslant 0$. Hence,

$$
C_{3} \geqslant 0 \quad \text { for } x \in \mathbb{R} \text {. }
$$

Next, we give an upper bound for $C_{3}$. To do this, we consider possible values of $x$ including $-k \leqslant x \leqslant 0$, $-1 \leqslant x \leqslant-k$ and $0 \leqslant x<k$.

If $-k \leqslant x<0$, then

$$
C_{3} \leqslant-x+k \leqslant|t|
$$

If $-1 \leqslant x \leqslant-k$, then $x^{2}-k^{2}>0$ and $k \leqslant 1$. By (13), we have

$$
\mathrm{e}^{x^{2} / 2-k^{2} / 2}=1+\left(\frac{x^{2}}{2}-\frac{k^{2}}{2}\right) \mathrm{e}^{x_{2}}
$$

for some $0 \leqslant x_{2} \leqslant \frac{x^{2}}{2}-\frac{k^{2}}{2}$. By the inequalities $-1 \leqslant x+$ $k \leqslant 0$ and $-1 \leqslant-t \leqslant x-k \leqslant 0$, we have $0 \leqslant x^{2}-k^{2} \leqslant$ $|t| \leqslant 1$. Hence,

$$
\begin{aligned}
C_{3} & =-x\left[1+\left(\frac{x^{2}}{2}-\frac{k^{2}}{2}\right) \mathrm{e}^{x_{2}}\right]+k \\
& \leqslant-x-\frac{\sqrt{\mathrm{e}} x|t|}{2}+x+t \\
& \leqslant 0.83|x||t|+|t| \quad \text { for }-1 \leqslant x \leqslant-k .
\end{aligned}
$$

Suppose that $0 \leqslant x<k$. Then, we have $x^{2}-k^{2}<0$. By (13), we have

$$
\mathrm{e}^{x^{2} / 2-k^{2} / 2}=1+\left(\frac{x^{2}}{2}-\frac{k^{2}}{2}\right) \mathrm{e}^{x_{3}}
$$

for some $x_{3}<0$. Note that $0 \leqslant k^{2}-x^{2}=(k-x)(k+x) \leqslant$ $t(2 x+t) \leqslant t(2 x+1)$. This implies that

$$
\begin{aligned}
C_{3} & =-x\left[1+\left(\frac{x^{2}}{2}-\frac{k^{2}}{2}\right) e^{x_{3}}\right]+k \\
& \leqslant-x+x\left(\frac{k^{2}}{2}-\frac{x^{2}}{2}\right) e^{x_{3}}+x+t \\
& \leqslant x\left(\frac{k^{2}}{2}-\frac{x^{2}}{2}\right)+t \\
& \leqslant x\left[\frac{t}{2}(2 x+1)\right]+t \\
& \leqslant x^{2}|t|+0.5|x||t|+|t| \quad \text { for } 0 \leqslant x<k .
\end{aligned}
$$

From (24)-(27), we have

$$
0 \leqslant C_{3} \leqslant x^{2}|t|+0.83|x||t|+|t| \text { for } k-t<x \leqslant k \text {. }
$$

Consequently,

$$
\left|f_{k}^{\prime}(x+t)-f_{k}^{\prime}(x)\right| \leqslant 2 x^{2}|t|+10.46|x||t|+12.16|t|
$$

for $k-t<x \leqslant k$.

From Cases 1-4, we can conclude that

$$
\left|f_{k}^{\prime}(x+t)-f_{k}^{\prime}(x)\right| \leqslant 2 x^{2}|t|+10.46|x||t|+12.16|t| .
$$

\section{PROOF OF THE MAIN THEOREM}

In this section, we use the Stein's method and property of the Stein solution to prove the main theorem.

Proof of Theorem 1: By modification of an argument in the proofs of Theorems 2.1-2.2 in [12] with the notation

$$
\begin{aligned}
\widehat{K}_{i}(t) & =Y_{i}\left[\mathbb{I}\left(-Y_{i} \leqslant t<0\right)-\mathbb{I}\left(0 \leqslant t \leqslant-Y_{i}\right)\right] \\
\text { and } \quad \widehat{K}(t) & =\sum_{i=1}^{l_{n}} \widehat{K}_{i}(t),
\end{aligned}
$$

we see that

$$
E W f_{k}(W)-E f_{k}^{\prime}(W)=R_{1}+R_{2}+R_{3},
$$

where $\quad R_{1}=E \int_{-\infty}^{\infty} f_{k}^{\prime}(W)[\widehat{K}(t)-E \widehat{K}(t)] \mathrm{d} t$,

$$
R_{2}=E \int_{|t|>1}\left[f_{k}^{\prime}(W+t)-f_{k}^{\prime}(W)\right] \widehat{K}(t) \mathrm{d} t,
$$

and

$$
R_{3}=E \int_{|t| \leqslant 1}\left[f_{k}^{\prime}(W+t)-f_{k}^{\prime}(W)\right] \widehat{K}(t) \mathrm{d} t .
$$


To bound $R_{1}, R_{2}$ and $R_{3}$, we note from [12] that

$$
\begin{gathered}
\int_{-\infty}^{\infty} \widehat{K}(t) \mathrm{d} t=\sum_{i=1}^{l_{n}} Y_{i}^{2}, \\
\int_{|t|>1} \widehat{K}_{i}(t) \mathrm{d} t \leqslant\left|Y_{i}\right|^{3},
\end{gathered}
$$

and $\quad \int_{|t| \leqslant 1}|t| \widehat{K}(t) \mathrm{d} t \leqslant \frac{1}{2} \sum_{i=1}^{l_{n}}\left|Y_{i}\right|\left(Y_{i}^{2} \wedge 1\right)$,

where $a \wedge b=\min \{a, b\}$ for $a, b \in \mathbb{R}$.

By (7) and (29), we obtain

$$
\begin{aligned}
\left|R_{1}\right| \leqslant\left\|f_{k}^{\prime}\right\| E\left|\sum_{i=1}^{l_{n}}\left(Y_{i}^{2}-E Y_{i}^{2}\right)\right| \\
\leqslant 0.8 E\left|\sum_{i=1}^{l_{n}}\left(Y_{i}^{2}-E Y_{i}^{2}\right)\right| .
\end{aligned}
$$

To bound $E\left|\sum_{i=1}^{l_{n}}\left(Y_{i}^{2}-E Y_{i}^{2}\right)\right|$, let $\xi_{i}=Y_{i} \mathbb{I}\left(\left|Y_{i}\right| \leqslant 1\right)$ for $i=1,2,3, \ldots, l_{n}$. Then, we can follow the proof of Theorem 2.2 in [12] to show that

$$
E\left|\sum_{i=1}^{l_{n}}\left(Y_{i}^{2}-E Y_{i}^{2}\right)\right| \leqslant\left(\operatorname{Var} \sum_{i=1}^{l_{n}} \xi_{i}^{2}\right)^{1 / 2}+2 \sum_{i=1}^{l_{n}} E\left|Y_{i}\right|^{3} .
$$

Since $Y_{i}^{\prime}$ s, $i=1,2,3, \ldots, l_{n}$, are independent,

$$
\operatorname{Var}\left(\sum_{i=1}^{l_{n}} \xi_{i}^{2}\right)=\sum_{i=1}^{l_{n}} \operatorname{Var} \xi_{i}^{2} \leqslant \sum_{i=1}^{l_{n}} E \xi_{i}^{4} \leqslant \sum_{i=1}^{l_{n}} E Y_{i}^{4} .
$$

By (32)-(34), we obtain

$$
\left|R_{1}\right| \leqslant 0.80\left(\sum_{i=1}^{l_{n}} E Y_{i}^{4}\right)^{1 / 2}+1.6 \sum_{i=1}^{l_{n}} E\left|Y_{i}\right|^{3} .
$$

Consider $R_{2}$. By (7) and (30), we have

$$
\left|R_{2}\right| \leqslant 1.6 \sum_{i=1}^{l_{n}} E \int_{|t|>1} \widehat{K}_{i}(t) \mathrm{d} t \leqslant 1.6 \sum_{i=1}^{l_{n}} E\left|Y_{i}\right|^{3} .
$$

Next, we consider $R_{3}$. By Proposition 1, we have $\left|f_{k}^{\prime}(x+t)-f_{k}^{\prime}(x)\right| \leqslant 2 x^{2}|t|+10.46|x||t|+12.16|t|$.

Hence,

$$
R_{3} \leqslant R_{3,1}+R_{3,2}+R_{3,3},
$$

where

$$
\begin{aligned}
& R_{3,1}=2 E \int_{|t| \leqslant 1} W^{2}|t| \widehat{K}(t) \mathrm{d} t, \\
& R_{3,2}=10.46 E \int_{|t| \leqslant 1}|W||t| \widehat{K}(t) \mathrm{d} t,
\end{aligned}
$$

and

$$
R_{3,3}=12.16 E \int_{|t| \leqslant 1}|t| \widehat{K}(t) \mathrm{d} t
$$

By (31) and the fact that

$$
\left(\sum_{i=1}^{d} a_{i}\right)^{k} \leqslant d^{k-1} \sum_{i=1}^{d} a_{i}^{k},
$$

for $a_{i}>0$ and $k, d \in \mathbb{N}$, we obtain

$$
\begin{aligned}
E\left(\int_{|t| \leqslant 1}|t| \widehat{K}(t) \mathrm{d} t\right)^{2} & \leqslant \frac{1}{4} E\left(\sum_{i=1}^{l_{n}}\left|Y_{i}\right|\left(Y_{i}^{2} \wedge 1\right)\right)^{2} \\
& \leqslant \frac{1}{4} E\left(\sum_{i=1}^{l_{n}}\left|Y_{i}\right|^{3}\right)^{2} \\
& \leqslant \frac{l_{n}}{4} \sum_{i=1}^{l_{n}} E Y_{i}^{6} .
\end{aligned}
$$

Hence,

$$
\begin{aligned}
& R_{3,1} \leqslant 2\left[E W^{4} E\left(\int_{|t| \leqslant 1}|t| \widehat{K}(t) \mathrm{d} t\right)^{2}\right]^{1 / 2} \\
& \leqslant\left(l_{n} E W^{4} \sum_{i=1}^{l_{n}} E Y_{i}^{6}\right)^{1 / 2} .
\end{aligned}
$$

By (31), we have

$$
\begin{aligned}
& R_{3,2} \leqslant 5.23 \sum_{i=1}^{l_{n}} E\left|W Y_{i}\right|\left(Y_{i}^{2} \wedge 1\right) \\
& R_{3,3} \leqslant 6.08 \sum_{i=1}^{l_{n}} E\left|Y_{i}\right|^{3} .
\end{aligned}
$$

We can use the argument in [12] to show that

$$
R_{3,2} \leqslant 15.69 \sum_{i=1}^{l_{n}} E\left|Y_{i}\right|^{3} .
$$

Hence, we conclude from (37)-(39) that

$$
R_{3} \leqslant\left(l_{n} E W^{4} \sum_{i=1}^{l_{n}} E Y_{i}^{6}\right)^{1 / 2}+21.77 \sum_{i=1}^{l_{n}} E\left|Y_{i}\right|^{3} .
$$

Combining (28), (35), (36), and (40), we obtain

$$
\begin{aligned}
\sup _{k>0}\left|E(W-k)^{+}-E(Z-k)^{+}\right| & \\
\leqslant 24.97 \sum_{i=1}^{l_{n}} E\left|Y_{i}\right|^{3} & +0.80\left(\sum_{i=1}^{l_{n}} E Y_{i}^{4}\right)^{1 / 2} \\
& +\left(l_{n} E W^{4} \sum_{i=1}^{l_{n}} E Y_{i}^{6}\right)^{1 / 2}
\end{aligned}
$$

Next, we want to bound the forth moment of $W$. By the local dependence condition, we have that $Y_{i}$ and 
$Y_{j}$ are independent for $i \neq j$. From this fact and $E Y_{i}=$ 0 , we have

$$
\begin{aligned}
\sum_{i=1}^{l_{n}} E Y_{i}^{2} & =\sum_{i=1}^{l_{n}} E Y_{i}^{2}+\sum_{i=1}^{l_{n}} \sum_{\substack{j=1 \\
j \neq i}}^{l_{n}} E Y_{i} Y_{j} \\
& =E\left(\sum_{i=1}^{l_{n}} Y_{i}\right)^{2}=E W^{2}=1 .
\end{aligned}
$$

Observe that $E Y_{j_{1}}^{3} Y_{j_{2}}=E Y_{j_{1}}^{2} Y_{j_{2}} Y_{j_{3}}=E Y_{j_{1}} Y_{j_{2}} Y_{j_{3}} Y_{j_{4}}=0$ for distinct indices $j_{i}$. Hence,

$$
\begin{aligned}
& E W^{4}=E\left(\sum_{i=1}^{l_{n}} Y_{i}\right)^{4} \\
& =\sum_{i=1}^{l_{n}} E Y_{i}^{4}+4 \sum_{j_{1}=1}^{l_{n}} \sum_{\substack{j_{2}=1 \\
j_{2} \neq j_{1}}}^{l_{n}} E Y_{j_{1}}^{3} Y_{j_{2}}+6 \sum_{j_{1}=1}^{l_{n}} \sum_{\substack{j_{2}=1 \\
j_{2}<j_{1}}}^{l_{n}} E Y_{j_{1}}^{2} Y_{j_{2}}^{2} \\
& +12 \sum_{j_{1}=1}^{l_{n}} \sum_{\substack{j_{2}=1 \\
j_{2} \neq j_{1}}}^{l_{n}} \sum_{\substack{j_{3}=1 \\
j_{3} \neq j_{1} \\
j_{3}<j_{2}}}^{l_{n}} E Y_{j_{1}}^{2} Y_{j_{2}} Y_{j_{3}} \\
& +24 \sum_{j_{1}=1}^{l_{n}} \sum_{\substack{j_{2}=1 \\
j_{2}<j_{1}}}^{l_{n} \sum_{3}<j_{2}} \sum_{j_{3}=1}^{l_{n}} \sum_{j_{4}=1}^{l_{n}} E Y_{j_{3}} Y_{j_{2}} Y_{j_{3}} Y_{j_{4}} \\
& \leqslant \sum_{i=1}^{l_{n}} E Y_{i}^{4}+3 \sum_{j_{1}=1}^{l_{n}} \sum_{\substack{j_{2}=1 \\
j_{2} \neq j_{1}}}^{l_{n}} E Y_{j_{1}}^{2} Y_{j_{2}}^{2} \\
& \leqslant \sum_{i=1}^{l_{n}} E Y_{i}^{4}+3\left(\sum_{i=1}^{l_{n}} E Y_{i}^{2}\right)^{2} \\
& \leqslant 3+\sum_{i=1}^{l_{n}} E Y_{i}^{4}
\end{aligned}
$$

Therefore, combining (41)-(42), we obtain Theorem 1 as required.

Acknowledgements: The first author is thankful for financial support by the Human Resource Development in Science Project (Science Achievement Scholarship of Thailand, SAST).

\section{REFERENCES}

1. Burtschell X, Gregory J, Laurent JP (2009) A comparative analysis of CDO pricing models under the factor copula framework. J Deriv 16, 9-37.

2. Buzková P, Teplý P (2012) Collateralized debt obligations valuation using the one factor Gaussian copula model. Prague Econ Pap 2012, 30-49.

3. Cao L, Jingqing Z, Lim K, Zhao Z (2008) An empirical study of pricing and hedging collateralized debt obligation (CDO). Adv Econom 22, 15-54.
4. Yang J, Hurd T, Zhang X (2006) Saddlepoint approximation method for pricing CDOs. $J$ Comput Financ 10, 1-20.

5. Pagés G, Wilbertz B (2012) Dual quantization for random walks with application to credit derivatives. $J$ Comput Financ 16, 33-60.

6. El Karoui N, Jiao Y (2009) Stein's method and zero bias transformation for CDO tranche pricing. Financ Stoch 13, 151-180.

7. El Karoui N, Jiao Y, Kurtz D (2008) Gaussian and Poisson approximation: application to CDOs tranche pricing. $J$ Comput Financ 2, 31-58.

8. Neammanee K, Yonghint N (2020) Poisson approximation for call function via Stein-Chen method. Bull Malaysian Math Sci Soc 43, 1135-1152.

9. Yonghint N, Neammanee K, Chaidee N (2020) Poisson approximation for locally dependent CDO. Commun Stat Theory Methods, 1-9.

10. Yonghint N, Neammanee K (2021) Refinement on Poisson approximation of CDOs. ScienceAsia 47, 388-392.

11. Jongpreechaharn S, Neammanee K (2019) Normal approximation for call function via Stein's method. Commun Stat Theory Methods 48, 3498-3517.

12. Chen LHY, Shao QM (2004) Normal approximation under local dependence. Ann Probab 32, 1985-2028.

13. Chen L, Röllin A (2013) Approximating dependent rare events. Bernoulli 19, 1243-1267.

14. Boutsikas MV, Eutichia Vaggelatou E (2010) A new method for obtaining sharp compound Poisson approximation error estimates for sums of locally dependent random variables. Bernoulli 16, 301-330.

15. Boutsikas MV (2006) Compound Poisson process approximation for locally dependent real-valued random variables via a new coupling inequality. Bernoulli 12, 501-514.

16. Adrian Röllin A (2008) Symmetric and centered binomial approximation of sums of locally dependent random variables. Electron $J$ Probab 13, 756-776.

17. Kumar AN (2021) Approximations to weighted sums of random variables. Bull Malaysian Math Sci Soc 44, $2447-2464$.

18. Fang X (2014) Discretized normal approximation by Stein's method. Bernoulli 20, 1404-1431.

19. Volgin AV (2017) Improving the rate of convergence in the multidimensional central limit theorem for sums of locally dependent random vectors. Prikl Diskretn Mat 36, 13-24. [in Russian]

20. Barbour AD, Xia A (2019) Multivariate approximation in total variation using local dependence. Electron J Probab 24, 1-35.

21. Stein C (1972) A bound for the error in the normal approximation to the distribution of a sum of dependent random variables. In: Lucien M, Cam L, Neyman J, Scott EL (eds) Proceedings of the Sixth Berkeley Symposium on Mathematical Statistics and Probability, vol 2, Berkeley, California, pp 583-602.

22. Chen LHY, Goldstein L, Shao QM (2010) Normal Approximation by Stein's Method, Springer Science \& Business Media, New York, NY.

23. Stein C (1986) Approximate Computation of Expectations, IMS Lecture Notes-Monograph Series Vol. 7, Hayward, CA. 